\title{
Posverdad del consumo de cannabis: de regreso a la medicina basada en la evidencia
}

\section{Post-truth Cannabis use: back to evidence-based medicine}

\author{
Hugo lópez-Pelayo*, laia Miquel De Montagut*, Cristina Casajuana Kögel*, \\ Mercè Balcells Oliveró*. \\ * Grup de Recerca en Addiccions Clínic, Hospital Clínic de Barcelona, IDIBAPS, \\ Universitat de Barcelona, Red de Trastornos adictivos (RETICS), Barcelona, Spain.
}

A unque la posverdad es un concepto de amplia difusión en el siglo XXI, ya en el año 1992 el dramaturgo Steve Tesich usó este término para referirse a la sociedad americana de su época de la siguiente manera "nosotros, como pueblo libre, libremente decidimos vivir en un mundo de posverdad" (Flood, 2016). En el año 2016 el Diccionario de Oxford le otorgó el premio de palabra del año y con él una definición clara y concisa "relativo a circunstancias en las que el hecho objetivo es menos influyente en la opinión pública que aquello que apela a la emoción o a las creencias personales" (Oxford English Living Dictionaries, 2017). Parece que la segunda mitad del siglo XX nos trajo la medicina basada en la evidencia y, el nuevo siglo nos obliga a defenderla. Ahora no se trata de encontrar la verdad a través del método científico sino de construir un relato y para ello explotar la ciencia como instrumento para edificarlo. Primero creamos un relato y luego buscamos la evidencia que lo sustenta. Es decir, la ciencia en algunos casos ha entrado de pleno en la posverdad. Tenemos algún ejemplo muy claro como la relación entre vacunas y autismo (Taylor, Swerdfeger y Eslick, 2014; Tomeny, Vargo y El-Toukhy, 2017)pero también alguno menos evidente como es el discurso social entorno al consumo de cannabis. Parece no importar que aún tengamos muchas más incógnitas que respuestas sobre las consecuencias -positivas y negativas- del consumo de cannabis sobre el individuo y la sociedad; lo más relevante es establecer una posición anti o pro.
En los últimos 20 años el consumo de cannabis ha experimentado un crecimiento elevado en las sociedades occidentales y en nuestro país en particular-del 4,6\% de usuarios en el último mes entre la población general (1997) al 7,3\% (2015). En paralelo al incremento del consumo regular de cannabis ha disminuido la percepción de riesgo sobre su uso regular. No sabemos si es causa o consecuencia pero más de $1 / 5$ parte de la población no cree que el consumo regular tenga un riesgo para la salud (Delegación del Gobierno para Plan Nacional sobre Drogas, 2015) y la sociedad se ha flexibilizado respecto al uso y la legalización (Sánchez Caballero, 2014). Probablemente el papel de la sociedad posmoderna ha influido en estos cambios, sobretodo, entre los más jóvenes (Blasco-Fontecilla, 2018).

\section{¿Qué sabemos y qué no sabemos del daño causado por el cannabis?}

El impacto sobre la salud mental está claramente establecido. Una excelente revisión de Robin Murray y colaboradores (2016) establece la relación entre el consumo de cannabis y la aparición de psicosis. Desmonta teorías con muy escasa evidencia científica como la de la auto-medicación o el impacto positivo del CBD sobre la salud mental de los usuarios, e incide en la importancia pronóstica del consumo de cannabis en pacientes con esquizofrenia. No se olvida de la importancia de diferenciar entre tipos de cannabis consumido (sintético, alta potencia, tradicional) e impacto sobre la psicopatología. Esta misma revisión

Recibido: Diciembre 2017; Aceptado: Marzo 2018

Enviar correspondencia a:

Mercè Balcells Oliveró. Unidad de Conducta Adictivas.

Hospital Clínic de Barcelona. G、Villarroel 170, 08036, Barcelona. 932275400. Email: mdbalcel@clinic.cat. 
hace referencia a otros aspectos de la salud mental que están relacionados con el consumo regular de cannabis como son la dependencia - 1 de cada 10 usuarios, hasta el $17 \%$ si se inicia en la adolescencia- o el daño cognitivo. Si que se mantiene más prudente al relacionar consumo de cannabis con trastornos afectivos y de ansiedad ( $\mathrm{Mu}-$ rray, Quigley, Quattrone, Englund y Di Forti, 2016). Por otra parte, un reciente meta-análisis muestra que el riesgo de psicosis se relaciona con la intensidad del consumo de cannabis (Marconi, Di Forti, Lewis, Murray y Vassos, 2016). Existen lagunas en cuanto a la relación del consumo regular de cannabis y los trastornos afectivos, ansiosos y el riesgo de suicidio. Podemos decir que la evidencia actual es insuficiente pero incipiente (Marconi et al., 2016). El daño cognitivo del uso de cannabis existe, es dosis-dependiente y parcialmente reversible con la cesación, y es mayor si el consumo aparece en la adolescencia. Por otra parte, parece que se confirma que el cannabis funciona como puerta de entrada a otras sustancias incluyendo lo que se ha denominado "puerta de entrada inversa", es decir, que también sería una vía de acceso al consumo de tabaco (Hall, 2014).

La salud física también se puede ver afectada por el consumo regular de cannabis. Donde la evidencia es más clara es sobre la salud cardiovascular, en cambio, el daño pulmonar y la capacidad carcinogénica es más dudosa. La capacidad carcinogénica del cannabis no está suficientemente documentada pero apunta como órganos diana a los pulmones, las vías respiratorias altas, esófago, testículo (tipo no seminona) y la vejiga. Una de las principales dificultades en estos estudios es el control de factores de confusión como el tabaco y en algunos casos la ausencia de estudios prospectivos (Hall, 2014). El síndrome de hiperémesis cannábica merece un capítulo aparte ya que es una entidad descrita sólo desde el año 2004 (Allen, de Moore, Heddle y Twartz, 2004) y actualmente á existen series de casos sobre la descripción clínica (Simonetto, Oxentenko, Herman y Szostek, 2012), un modesto estudio sobre prevalencia (Bruguera, López-Pelayo, Miquel y Balcells-Oliveró, 2016) y alguna investigación aislada sobre su tratamiento (Pélissier, Claudet, Gandia-Mailly, Benyamina y Franchitto, 2016; Sorensen, DeSanto, Borgelt, Phillips y Monte, 2017). La afectación fetal del consumo de cannabis durante el embarazo no está del todo clara. Sin embargo, algunos estudios sugieren que afectaría en forma de bajo peso al nacer e incremento del riesgo de parto pretérmino. Otros efectos posibles del consumo durante el embarazo son el menor rendimiento cognitivo en la adolescencia -incluyendo el coeficiente intelectual-, alteraciones conductuales, delincuencia, menor rendimiento académico y trastornos afectivos(Hall, 2014).

El impacto psicosocial, sobre todo entre los adolescentes y adultos jóvenes, también tiene un soporte bibliográfico contundente. Aunque la evidencia científica resulta contradictoria cuando nos referimos al riesgo de fracaso escolar ya que aunque la asociación es clara, la causalidad de la misma está en duda debido a que algunos estudios sugieren que el consumo de cannabis y el fracaso escolar comparten factores de riesgo preexistentes (Lynskey y Hall, 2000). No debemos olvidar que las asociación entre accidentes de tráfico y conducir bajo los efectos del cannabis es clara; concretamente el riesgo de accidente se duplica o incluso triplica si se conduce intoxicado. Esta asociación tiene una base neurocognitiva en la disminución del tiempo de reacción, la capacidad de procesamiento de la información, la coordinación perceptivo-motora, el rendimiento motor, la atención y la conducta de seguimiento(Hall, 2014).

Es importante recordar que la falta de evidencia no es evidencia de que no hay relación. Al mismo tiempo, cuando un mismo tema de estudio presenta evidencia mixta tampoco podemos establecer relaciones claras de causa-efecto. Tampoco si los criterios científicos de causalidad no están presentes: validez interna (fuerza de asociación, efecto dosis-respuesta, secuencia temporal) y coherencia científica (consistencia, plausibilidad biológica, especificad de asociación y analogía, evidencia experimental) (Hill, 1965).

En base a la bibliografía existente, la guía clínica "LowerRisk Cannabis Use Guidelines" recomienda con un grado de evidencia sustancial: 1) la mejor manera de evitar los riesgos asociados al consumo de cannabis es la abstinencia; 2) los mensajes de prevención deben enfatizar el retraso en la edad de inicio del consumo ya que los riesgos de efectos adversos son menores si el consumo se inicia más tardíamente; 3) los usuarios de cannabis deberían conocer la concentración de THC y CBD intentar evitar los de una alta concentración de THC (Casajuana, López-Pelayo, Balcells, Colom y Gual, 2018) y priorizar los de bajo ratio THC:CBD ya que los efectos adversos se relacionan con la alta dosis de THC -las plantas que producen elevadas concentraciones de THC generan concentraciones bajas de CBD (Murray et al., 2016); 4) el cannabis en combustión y/o mezclado con tabaco se debería evitar priorizando otras formas de consumo (vaporizado o comestible); 5) el consumo regular o diario es el que está asociado a la mayoría de efectos adversos. Los usuarios deberían intentar mantener un consumo ocasional (una vez a la semana, fin de semana o menor frecuencia); 6) los usuarios deberían evitar conducir durante las 6 horas posteriores al consumo -siempre que esto no contradiga la legislación local vigente- y nunca conducir tras ingerir alcohol y cannabis ya que existen potenciación de los efectos agudos negativos sobre la capacidad de conducción. Evitar la conducción como mínimo las 6 horas tras la ingesta se basa en que el pico plasmático de THC es a los 5-30 minutos tras su uso y disminuye a las 2-4 horas aunque la intoxicación y el deterioro cognitivo agudo pueden persistir entre 3 y 6 horas. Aún podría alargarse más en caso de elevadas dosis de THC y/o ingesta por vía digestiva. Con menor grado de evidencia también recomienda: 1) Los usuarios deberían 
evitar el cannabis sintético por el riesgo de efectos agudos adversos. El cannabis sintético tiene elevada afinidad por receptores CB1, es un agonista total a diferencia del cannabis tradicional que es parcial y se ha informado de diversas complicaciones incluyendo psicosis aguda, fallo renal, infarto de miocardio, agresividad e isquemia cerebral. Hasta 24 casos de muertes se han relacionado con el uso de cannabis sintético (Gurney, Scott, Kacinko, Presley y Logan, 2014; Tournebize, Gibaja y Kahn, 2017); 2) La inhalación profunda debería ser evitada para reducir el riesgo de daño pulmonar; 3) Evitar la combinación de dos o más conductas de riesgo descritas anteriormente. Más allá del riesgo individual relacionado con el patrón de consumo, existen poblaciones de riesgo ante cualquier tipo de consumo de cannabis: historia personal o familiar de psicosis, embarazadas y lactantes, y enfermos con problemas cardiovasculares. Aunque sin evidencia suficiente, por un principio de prudencia también deberían evitar cualquier consumo de cannabis las personas con historia personal o familiar de otros trastornos mentales (Fischer et al., 2017).

Existe una elevada y creciente demanda de tratamiento para la dependencia del cannabis (Mounteney et al., 2016), pese a ello, sabemos que el impacto del tratamiento es limitado, ya que se logran tasas de abstinencia bajas (Hall, 2017), si bien, una reducción en el consumo y una mejora en la calidad de vida si se consiguen (Gates, Sabioni, Copeland, Le Foll y Gowing, 2016a). La combinación de la entrevista motivacional, la terapia cognitivo-conductual y la inclusión de incentivos por la abstinencia deben ser la base de cualquier tratamiento (Carney, Myers, Louw y Okwundu, 2016; Denis, Lavie, Fatseas and Auriacombe, 2006; Gates, Sabioni, Copeland, Le Foll y Gowing, 2016b). En cuanto a los fármacos, la Gabapentina y la N-acetilcisteina son prometedores aunque la evidencia aún es débil (Marshall, Gowing, Ali y Le Foll, 2014).

Por último, la legalización del cannabis puede tener un impacto sobre el precio del mismo (reducción), la percepción social (normalización del uso), sobre la disponibilidad (mayor accesibilidad) y sobre la potencia (incremento). Esto podría traducirse en un incremento del uso a largo plazo de magnitud desconocida (Álvarez, Gamella y Parra, 2017; Hall, 2017). En este sentido, los expertos de ALICERAP (www.alicerap.eu) apuntan que las medidas extremas (prohibiciones o liberalización absoluta) no son una buena estrategia pero que la regularización del uso de cannabis puede ser beneficiosa en términos de salud pública con el fin de evitar consecuencias indeseadas del prohibicionismo como son el crimen organizado y la violencia, el estigma y la discriminación de los usuarios, el uso en la vía pública de las sustancias y la pérdida de derechos civiles, empleo, hogar y relaciones personales de los usuario (Apfel, 2013). Sin embargo, estas hipótesis tienen que ser evaluadas regularmente, sobre todo cuando ya hay indicios que en USA, tras la legalización, se ha incrementado el uso, la exposición accidental en menores de edad (Wang et al., 2016) y la percepción de riesgo ha disminuido (Schuermeyer et al., 2014; Sobesky y Gorgens, 2016). En este sentido los indicadores de potencia, dosis, ventas de cannabis, producción legal y daños asociados al cannabis (accidentes de tráfico, visitas a urgencias, atención especializada del trastorno por uso de cannabis, prevalencia del consumo en pacientes de salud mental y justicia) deben ser tenidos en cuenta (Hall, 2017). Por último, probablemente unas políticas públicas basadas en las Three Best Buys (limitación de acceso a la sustancia, limitación de la publicidad e incremento del precio a través de medidas impositivas o precio mínimo por unidad de consumo) (Baccini y Carreras, 2014; Matrai et al., 2014) tendría sentido para aproximarnos al modelo contemporáneo de prevención del tabaquismo y alejarnos del modelo poco eficaz y heterogéneo de prevención de las consecuencias del consumo de alcohol. Esto quiere decir evitar poner en manos de la naciente industria del cannabis las medidas de prevención bajo el lema "consumo responsable" y asumir estrategias de prevención basadas en la evidencia.

Pese a todo, cabe huir del alarmismo. El cannabis es una sustancia menos deletérea que otras como el alcohol o la nicotina. Por ejemplo, Lachenmeier y Rhem (2015) utilizan el concepto Margin of Exposure (MOE) para realizar una evaluación comparativa del riesgo de varias sustancias. El MOE es el ratio entre el umbral tóxico y la estimación del consumo humano; se calcula en base a la dosis letal media en animales de experimentación y estudios individuales y poblacionales. El MOE estimado para el uso diario del THC es más favorable que para el resto de sustancias como las benzodiacepinas, los estimulantes (cocaína, anfetamina y metanfetamina), el MDMA, la nicotina, la heroína y el alcohol (Lachenmeier y Rehm, 2015).

\section{¿Qué sabemos y no sabemos realmente del beneficio del cannabis?}

El uso del cannabis en su forma natural o como preparado farmacéutico (dronabinol) se postula como tratamiento en diversas condiciones médicas. A día de hoy, no existe suficiente evidencia de eficacia ni seguridad para considerarlo una opción terapéutica para mejorar el apetito, incrementar el peso o el ánimo en pacientes con VIH/ SIDA ya que los estudios son de corta duración y con muestras pequeñas que no consideran los efectos a largo plazo (Lutge, Gray y Siegfried, 2013). Tampoco parece tener un rol en tratar la fibromialgia (Walitt, Klose, Fitzcharles, Phillips y Häuser, 2016) ni las alteraciones conductuales de la demencia (Krishnan, Cairns y Howard, 2009). Como antiemético en niños y jóvenes con patología oncológica tiene una escasa evidencia, existen mejores alternativas con un perfil de efectos adversos preferible (Phillips et al., 2016). En cambio en adultos con emesis asociada a quimioterapia puede ser una alternativa cuando se trate de 
una sintomatología refractaria. Esta conclusión podría modificarse en un futuro próximo con la aparición de nuevos antieméticos más seguros (Smith, Azariah, Lavender, Stoner y Bettiol, 2015). El cannabidiol (CBD) podría tener un impacto positivo sobre la epilepsia refractaria pero aún es pronto para concluirlo (Gloss y Vickrey, 2014) y además la presencia de este compuesto en los preparados naturales es mínima(Casajuana Kögel et al., 2017), por tanto no podemos recomendar su uso en este contexto. Aunque parece que el cannabis podría ser eficaz para el dolor secundario a la artritis reumatoide, su seguridad clínica no está suficientemente comprobada, existiendo casos descritos de psicosis e ideación suicida (Richards, Whittle y Buchbinder, 2012). Tampoco en cuanto el dolor crónico asociado al cáncer el balance riesgo beneficio está claro aunque la eficacia parezca suficiente (Blake et al., 2017).

Un reciente análisis de más de 10000 estudios científicos por parte de la "US National Academies of Science, Engineering, and Medicine” ha concluido que la evidencia para su uso medicinal á es concluyente en el dolor crónico, algunos síntomas de la esclerosis múltiple y como antiemético post-quimioterapia en el adulto (Washington, 2017).

Finalmente, cabe recordar que el objetivo de la medicina es mejorar la calidad de vida y en este sentido la evidencia del cannabis medicinal es inconcluyente en el mejor de los casos, cuando no negativa (por ejemplo en los pacientes que padecen VIH) (Goldenberg, Reid, IsHak y Danovitch, 2017).

En todo caso, para poder ser usado como un medicamento debería seguir las exigencias de seguridad y evidencia que se pide a cualquier nuevo fármaco.

\section{¿Qué información nos falta para tomar decisiones adecuadas?}

En un momento en el que la evidencia científica muestra que el consumo regular de cannabis puede tener efectos adversos considerables, su uso medicinal es aún limitado y la legislación parece virar desde el prohibicionismo a la liberalización debemos promover una regularización basada en la evidencia. Carecemos de herramientas para la prevención en un marco de regularización que sí están disponibles en el caso del alcohol o tabaco. Pese a que las poblaciones de riesgo específico están bien definidas (embarazadas, jóvenes, antecedentes familiares o personales de trastorno psicótico, etc.), no disponemos de una definición operativa de consumo de riesgo basada en patrones de consumo (cantidad y frecuencia). Sin embargo, tenemos como punto de partida la definición conceptual de la OMS del consumo de riesgo de sustancias: "un patrón de uso que incrementa el riesgo de consecuencias dañinas para el usuario (salud física, mental o consecuencias sociales). En contraste con el consumo problemático, el consumo de riesgo tiene un significado en la salud pública más allá de la ausencia de ningún trastorno actual en el usuario a nivel individual" (WHO, 2017). En el consumo de alcohol podemos identificar como consumo de riesgo aquel usuario que ingiere 28 Unidades de Bebida Estándar a la semana siendo hombre o 17 siendo mujer. Además ha permitido crear un instrumento de cribado rápido, fiable y eficaz como es el AUDIT-C (Gual, Segura, Contel, Heather y Colom, 2002). Por el momento no ha podido llevarse a cabo una definición de este tipo ante la ausencia de estandarización en el registro del consumo de cannabis. Recientemente, hemos publicado una investigación que estandariza el consumo a través de las Unidades de Porro Estándar (UPE, en inglés Standard Joint Unit) y en el que cada UPE equivale a $7 \mathrm{mg}$ de THC (Casajuana et al., 2017; Casajuana Kögel et al., 2017). Este estudio debería ser reproducido en otras regiones y culturas, y adaptarse como se hizo la Unidad de Bebida Estándar. El siguiente paso lógico es el establecimiento de un patrón de riesgo en función de la cantidad y frecuencia a través de su correlación con las múltiples dimensiones de los daños asociados al consumo de cannabis. Aunque este objetivo es muy ambicioso es el paso necesario para que las políticas de intervención se focalicen en los grupos de mayor riesgo, sean precoces y breves, y consecuentemente eficientes.

Por otra parte, creemos necesario la creación de redes de trabajo para la identificación e intervención precoz del consumo de riesgo de cannabis como la propuesta por Socidrogalcohol a través de Cannared. Su misión principal es la de formar a profesionales en estrategias de prevención primaria y secundaria en el consumo de cannabis que hayan demostrado suficiente evidencia para ser difundidas.

En conclusión, en la era de las posverdad es más importante que nunca que los profesionales (científicos, asistenciales, docentes, etc.) tengamos información fiable y actualizada sobre los riesgos y beneficios del consumo regular de cannabis. Para ello es fundamental tener herramientas de registro y definiciones operativas del consumo de riesgo de cannabis.

\section{Conflicto de intereses}

Hugo López-Pelayo ha recibido honorarios y becas de viaje de Lundbeck, Lilly, Janssen, Pfizer, Rovi, Esteve. Laia Miquel y María Mercedes Balcells han recibido honorarios de Lundbeck. Los demás autores declaran no tener ningún conflicto de intereses potencial. Los honorarios mencionados no influyeron en este artículo.

\section{Referencias}

Allen, J. H., de Moore, G. M., Heddle, R. y Twartz, J. C. (2004). Cannabinoid hyperemesis: cyclical hyperemesis in association with chronic cannabis abuse. Gut, 53, 1566-1570. doi:10.1136/gut.2003.036350.

Álvarez, A., Gamella, J. F. y Parra, I. (2017). La legalización de los derivados del cannabis en España: Hipótesis sobre un potencial mercado emergente. Adicciones, 29, 195-206. doi:10.20882/adicciones.807. 
Apfel, F. (2013). Cannabis - from prohibition to regulation "When the music changes so does the dance." Recuperado de http://www.alicerap.eu/.

Baccini, M. y Carreras, G. (2014). Analyzing and Comparing the Association Between Control Policy Measures and Alcohol Consumption in Europe. Substance Use E Misuse, 49, 1684-1691. doi:10.3109/10826084.2014.914373.

Blake, A., Wan, B. A., Malek, L., DeAngelis, C., Diaz, P., Lao, N., ... O'Hearn, S. (2017). A selective review of medical cannabis in cancer pain management. Annals of Palliative Medicine, 6, 805-805. doi:10.21037/apm.2017.08.05.

Blasco-Fontecilla, H. (2018). Posmodernidad, sociedades adictivas, cannabis y comportamiento suicida: ¿Hacia un mundo feliz? Adicciones, 30, 3-8. doi:10.20882/adicciones.1104.

Bruguera, P., López-Pelayo, H., Miquel, L. y Balcells-Oliveró, M. (2016). High prevalence of cannabinoid hyperemesis syndrome in marijuana users. Emergencias, 28, 249-251.

Carney, T., Myers, B. J., Louw, J. y Okwundu, C. I. (2016). Brief school-based interventions and behavioural outcomes for substance-using adolescents. In T. Carney (Ed.), Cochrane Database of Systematic Reviews (p. CD008969). Chichester, UK: John Wiley \& Sons, Ltd. doi:10.1002/14651858.CD008969.pub3.

Casajuana, C., López-Pelayo, H., Balcells, M. M., Colom, J. y Gual, A. (2018). Constituyentes psicoactivos del cannabis y sus implicaciones clínicas: una revisión sistemática. Adicciones, 30, 140-151. doi:10.20882/adicciones.858.

Casajuana, C., López-Pelayo, H., Mercedes Balcells, M., Miquel, L., Teixidó, L., Colom, J. y Gual, A. (2017). Estableciendo la unidad de porro estándar: estudio piloto. Adicciones, 29, 227-232. doi:10.20882/adicciones.721.

Casajuana Kögel, C., Balcells-Olivero, M. M., López-Pelayo, H., Miquel, L., Teixidó, L., Colom, J., ... Gual, A. (2017). The Standard Joint Unit. Drug and Alcohol Dependence, 176, 109-116. doi:10.1016/j.drugalcdep.2017.03.010.

Delegación del Gobierno para Plan Nacional sobre Drogas. (2015). Encuesta sobre alcohol y drogas en población general España EDADES 2015-2016.

Denis, C., Lavie, E., Fatseas, M. y Auriacombe, M. (2006). Psychotherapeutic interventions for cannabis abuse and/or dependence in outpatient settings. In C. Denis (Ed.), Cochrane Database of Systematic Reviews (p. CD005336). Chichester, UK: John Wiley \& Sons, Ltd. doi:10.1002/14651858.CD005336.pub2.

Fischer, B., Russell, C., Sabioni, P., van den Brink, W., Le Foll, B., Hall, W., ... Room, R. (2017). Lower-Risk Cannabis Use Guidelines: A Comprehensive Update of Evidence and Recommendations. American Journal of Public Health, 107, 1277-1277. doi:10.2105/AJPH.2017.303818a.

Flood, A. (2016). Post-truth' named word of the year by Oxford Dictionaries. The Guardian. Recuperado de ht- tps://www.theguardian.com/books/2016/nov/15/posttruth-named-word-of-the-year-by-oxford-dictionaries.

Gates, P. J., Sabioni, P., Copeland, J., Le Foll, B. y Gowing, L. (2016a). Psychosocial interventions for cannabis use disorder. In P. J. Gates (Ed.), Cochrane Database of Systematic Reviews (p. CD005336). Chichester, UK: John Wiley \& Sons, Ltd. doi:10.1002/14651858.CD005336.pub4.

Gates, P. J., Sabioni, P., Copeland, J., Le Foll, B. y Gowing, L. (2016b). Psychosocial interventions for cannabis use disorder. The Cochrane Database of Systematic Reviews, (5), CD005336. doi:10.1002/14651858.CD005336.pub4.

Gloss, D. y Vickrey, B. (2014). Cannabinoids for epilepsy. In D. Gloss (Ed.), Cochrane Database of Systematic Reviews (p. CD009270). Chichester, UK: John Wiley \& Sons, Ltd. doi:10.1002/14651858.CD009270.pub3.

Goldenberg, M., Reid, M. W., IsHak, W. W. y Danovitch, I. (2017). The impact of cannabis and cannabinoids for medical conditions on health-related quality of life: A systematic review and meta-analysis. Drug and Alcohol Dependence, 174, 80-90. doi:10.1016/j.drugalcdep.2016.12.030.

Gual, A., Segura, L., Contel, M., Heather, N. y Colom, J. (2002). AUDIT-3 and AUDIT-4: effectiveness of two short forms of the Alcohol Use Disoders Identification Test. Alcohol and Alcoholism, 37, 591-596.

Gurney, S. M. R., Scott, K. S., Kacinko, S. L., Presley, B. C. y Logan, B. K. (2014). Pharmacology, toxicology, and adverse effects of synthetic cannabinoid drugs. Forensic Science Review, 26, 54-76.

Hall, W. (2014). What has research over the past two decades revealed about the adverse health effects of recreational cannabis use? Addiction, 110, 19-35. doi:10.1111/ add.12703.

Hall, W. (2017). Alcohol and cannabis: Comparing their adverse health effects and regulatory regimes. The International Journal on Drug Policy, 42, 57-62. doi:10.1016/j. drugpo.2016.10.021.

Hill, A. B. (1965). The environment and disease: association or causation? Proceedings of the Royal Society of Medici$n e, 58,295-300$.

Krishnan, S., Cairns, R. y Howard, R. (2009). Cannabinoids for the treatment of dementia. In S. Krishnan (Ed.), Cochrane Database of Systematic Reviews (p. CD007204). Chichester, UK: John Wiley \& Sons, Ltd. doi:10.1002/14651858.CD007204.pub2.

Lachenmeier, D. W. y Rehm, J. (2015). Comparative risk assessment of alcohol, tobacco, cannabis and other illicit drugs using the margin of exposure approach. Scientific Reports, 5, 8126. doi:10.1038/srep08126.

Lutge, E. E., Gray, A. y Siegfried, N. (2013). The medical use of cannabis for reducing morbidity and mortality in patients with HIV/AIDS. In E. E. Lutge (Ed.), Cochrane Database of Systematic Reviews (p. CD005175). Chichester, UK: John Wiley \& Sons, Ltd. doi:10.1002/14651858. CD005175.pub3. 
Lynskey, M. y Hall, W. (2000). The effects of adolescent cannabis use on educational attainment: a review. Addiction (Abingdon, England), 95, 1621-1630.

Marconi, A., Di Forti, M., Lewis, C. M., Murray, R. M. y Vassos, E. (2016). Meta-analysis of the Association Between the Level of Cannabis Use and Risk of Psychosis. Schizophrenia Bulletin, 42, 1262-1269. doi:10.1093/schbul/sbw003.

Marshall, K., Gowing, L., Ali, R. y Le Foll, B. (2014). Pharmacotherapies for cannabis dependence. In $\mathrm{L}$. Gowing (Ed.), Cochrane Database of Systematic Reviews (p. CD008940). Chichester, UK: John Wiley \& Sons, Ltd. doi:10.1002/14651858.CD008940.pub2.

Matrai, S., Casajuana, C., Allamani, A., Baccini, M., Pepe, P., Massini, G. y Gual, A. (2014). The relationships between the impact of alcoholic beverage control policies, selected contextual determinants, and alcohol drinking in Spain. Substance Use E Misuse, 49, 1665-1683. doi:10. 3109/10826084.2014.913398.

Mounteney, J., Griffiths, P., Sedefov, R., Noor, A., Vicente, J. y Simon, R. (2016). The drug situation in Europe: an overview of data available on illicit drugs and new psychoactive substances from European monitoring in 2015. Addiction, 111, 34-48. doi.org/10.1111/ add.13056.

Murray, R. M., Quigley, H., Quattrone, D., Englund, A. y Di Forti, M. (2016). Traditional marijuana, high-potency cannabis and synthetic cannabinoids: increasing risk for psychosis. World Psychiatry, 15, 195-204. doi:10.1002/ wps.20341.

Oxford English Living Dictionaries. (2017). Recuperado de https://en.oxforddictionaries.com/definition/post-truth.

Pélissier, F., Claudet, I., Gandia-Mailly, P., Benyamina, A. y Franchitto, N. (2016). Cannabis Hyperemesis Syndrome in the Emergency Department: How Can a Specialized Addiction Team Be Useful? A Pilot Study. The Journal of Emergency Medicine, 51, 544-551. doi:10.1016/j.jemermed.2016.06.009.

Phillips, R. S., Friend, A. J., Gibson, F., Houghton, E., Gopaul, S., Craig, J. V y Pizer, B. (2016). Antiemetic medication for prevention and treatment of chemotherapy-induced nausea and vomiting in childhood. In R. S. Phillips (Ed.), Cochrane Database of Systematic Reviews (Vol. 2, p. CD007786). Chichester, UK: John Wiley \& Sons, Ltd. doi:10.1002/14651858.CD007786.pub3.

Richards, B. L., Whittle, S. L. y Buchbinder, R. (2012). Neuromodulators for pain management in rheumatoid arthritis. In B. L. Richards (Ed.), Cochrane Database of Systematic Reviews (Vol. 1, p. CD008921). Chichester, UK: John Wiley \& Sons, Ltd. doi:10.1002/14651858. CD008921.pub2.

Sánchez Caballero, D. (2014). Cada vez más jóvenes españoles apoyan la legalización del cannabis. Eldiario.es. Recuperado de http://www.eldiario.es/sociedad/Quemando-etapas-regulacion-cannabis_0_296070879.html.
Schuermeyer, J., Salomonsen-Sautel, S., Price, R. K., Balan, S., Thurstone, C., Min, S.J. y Sakai, J. T. (2014). Temporal trends in marijuana attitudes, availability and use in Colorado compared to non-medical marijuana states: 2003-11. Drug and Alcohol Dependence, 140, 145-155. doi:10.1016/j.drugalcdep.2014.04.016.

Simonetto, D. A., Oxentenko, A. S., Herman, M. L. y Szostek, J. H. (2012). Cannabinoid hyperemesis: a case series of 98 patients. Mayo Clinic Proceedings, 87, 114-119. doi:10.1016/j.mayocp.2011.10.005.

Smith, L. A., Azariah, F., Lavender, V. T., Stoner, N. S. y Bettiol, S. (2015). Cannabinoids for nausea and vomiting in adults with cancer receiving chemotherapy. In L. A. Smith (Ed.), Cochrane Database of Systematic Reviews (p. CD009464). Chichester, UK: John Wiley \& Sons, Ltd. doi:10.1002/14651858.CD009464.pub2.

Sobesky, M. y Gorgens, K. (2016). Cannabis and adolescents: Exploring the substance misuse treatment provider experience in a climate of legalization. International Journal of Drug Policy, 33, 66-74. doi:10.1016/j. drugpo.2016.02.008.

Sorensen, C. J., DeSanto, K., Borgelt, L., Phillips, K. T. y Monte, A. A. (2017). Cannabinoid Hyperemesis Syndrome: Diagnosis, Pathophysiology, and Treatment-a Systematic Review. Journal of Medical Toxicology, 13, 71-87. doi:10.1007/s13181-016-0595-z.

Taylor, L. E., Swerdfeger, A. L. y Eslick, G. D. (2014). Vaccines are not associated with autism: An evidence-based meta-analysis of case-control and cohort studies. Vaccine, 32, 3623-3629. doi:10.1016/j.vaccine.2014.04.085.

Tomeny, T. S., Vargo, C. J. y El-Toukhy, S. (2017). Geographic and demographic correlates of autism-related anti-vaccine beliefs on Twitter, 2009-15. Social Science $\mathcal{E}$ Medicine, 191, 168-175. doi:10.1016/j.socscimed.2017.08.041.

Tournebize, J., Gibaja, V. y Kahn, J.-P. (2017). Acute effects of synthetic cannabinoids: Update 2015. Substance Abuse, 38, 344-366. doi:10.1080/08897077.2016.1219438.

Walitt, B., Klose, P., Fitzcharles, M.-A., Phillips, T. y Häuser, W. (2016). Cannabinoids for fibromyalgia. The Cochrane Database of Systematic Reviews, 7, CD011694. doi:10.1002/14651858.CD011694.pub2.

Wang, G. S., Le Lait, M.-C., Deakyne, S. J., Bronstein, A. C., Bajaj, L. y Roosevelt, G. (2016). Unintentional Pediatric Exposures to Marijuana in Colorado, 2009-2015. JAMA Pediatrics, 170, e160971. doi:10.1001/jamapediatrics.2016.0971.

Washington, D. T. N. A. P. (Ed.). (2017). The Health Effects of Cannabis and Cannabinoids: The Current State of Evidence and Recommendations for Research. doi:10.17226/24625.

World Health Organization. (2017). Management of substance abuse. Recuperado de http://www.who.int/substance_abuse/terminology/definition3/en/. 